\title{
Case Report of Advanced Childhood Nasopharyngeal Carcinoma: Is Radiotherapy Dose Deescalation the Right Way in Cood Responders to Induction Chemotherapy
}

\author{
Encheva E. ${ }^{1}$, Ivanovska Hr. ${ }^{1}$, Iliev G. ${ }^{2}$, Bochev P. ${ }^{3}$, Chaushev B. ${ }^{3}$, \\ Yordanova Ts. ${ }^{3}$, Georgiev. R. ${ }^{4}$, Kaleva V. ${ }^{5}$, Hristozova Hr. ${ }^{5}$, Milkov M. ${ }^{6}$ \\ 'Radiotherapy Department, University Hospital "St. Marina" - Varna \\ ${ }^{2}$ ENT Department, University Hospital "St. Marina" - Varna \\ ${ }^{3}$ Nuclear Medicine Department, University Hospital "St. Marina" - Varna \\ ${ }^{4}$ Imaging Department, University Hospital "St. Marina" - Varna \\ ${ }^{5}$ Pediatric Oncohematology Department, University Hospital "St. Marina" - Varna \\ ${ }^{6}$ Faculty of Dental Medicine, Medical University Varna
}

\begin{abstract}
Objectives:. Treatment of childhood NPC similar to adults consists of radiotherapy and chemotherapy, but distant failure is often observed, which led to introducing the induction chemotherapy followed by radiation or chemoradiation. The improved survival rates raised the question of late toxicity. The options for lowering the toxicity rate is the application of advanced radiotherapy techniques like IMRT and VMAT, and deescalation of the radiation dose in good responders and early NPC.

Case report: We report a case of 13 -years old male patient with a high-risk childhood undifferentiated NPC, stage cT4 cN2b M0. He presented with unilateral swallowing at the middle third ofleft muscle sterenocleidomastoideus, and headache, fever, sore throat and intermittent nasal bleeding for an year. Diagnostic MRI and PET/CT showed good concordance for primary tumor extension and lymph node involvement. Three coursesinduction chemotherapy were applied according to NPC2003-GPOH protocolwith good treatment response. The restaging PET/CT found no distant metastasis. Deescalatedprotocol of radiotherapy alone was delivered to 50.4 Gy total dose with IGRT, VMAT irradiation technique. At three month PET/CT follow up a solitary bone lesion was detected

Conclusion: The present case proved that in high risk patients more aggressive treatment strategies should be recommended with no omission of concurrent chemotherapy even after full response. Deescalation of radiotherapy dose probably is not appropriate in this group of patients. MRI and PET CT should be used as complementary imaging modalities for early detection of locoregional or distant metastasis.
\end{abstract}

Key words: Childhood nasopharyngeal carcinoma, treatment, radiotherapy, deescalation radiation dose, VMAT

\section{Introduction}

The incidence of nasopharyngeal carcinoma (NPC) in children worldwide is low. Most of the children are diagnosed with undifferentiated NPC in advanced stage - locoregional or metastatic [1]. Childhood NPC is very rare disease and in Bulgaria. According to the Bulgarian National Cancer Registry for the years 2014-2015 only one case in the age range $0-14$ years is recorded and it was a male patient [2].

Treatment of childhood NPC similar to adults consists of radiotherapy and chemotherapy. Different regimens have been explored like single modality radiotherapy, combined modality or consequential treatment [3]. High dose radiotherapy alone in children achieves $30-60 \%$ cure $[1,3]$.Distant failure is often observed in children, which led to introducing the induction chemotherapy followed by radiation or chemoradiation $[4,5]$. The reported 5 year survival rates reached $70-90 \%$ [6]. Improving cure rates with multimodality therapy raises the question of late toxicity [6].One way of lowering the toxicity rate is the application of modern radiotherapy techniques like IMRT and VMAT. Another option is the deescalation of the radiation dose in good responders and early NPC [4-6]. 
There are no clear international consensus to guide the daily clinical practice, especially in countries with low incidence of childhood NPC.

The aim of the present article is to report the first case of childhood NPC, treated at Radiotherapy Department, University Hospital Saint Marina, Varna, Bulgaria.

\section{Case presentation}

A 13-year-old male child presented with unilateral swallowing at the middle third ofleft muscle sterenocleidomastoideus, which was preceded by complains ofheadache, fever, sore throat and intermittent nasal bleeding for an year. At outpatient basis different antibiotics treatment was given with temporary or no effect. In march 2017 CT enhanced scan of head and neck region revealed left retrobulbar tumor formation with infiltration of ethmoidal and sphenoidal sinuses and nasopharynx. The ophthalmological exam revealed left exophthalmos with preserved vision. At ENT physical examination found a polypoid mass of nasopharynx. A biopsy of the formation confirmed undifferentiated carcinoma, with positive immunohistochemistry for CK5/6, and negative for CK7 expression. Further imaging studies were performed -MRI of head and neck and FDG PET/CT and the tumor was staged cT4cN2bcM0. The MRI examination showed tumor formation in nasopharynx, with expansion to sphenoid sinus, left ethmoidal cells, middle nasal cavity, left orbit and left parapharyngeal space. In the left orbit the tumor dislocated in upper direction the lower straight and obliquemuscles and the optic nerve. Bilateral lymph node involvement with biggest mass described at right level IIb 37x 20mm was seen (Fig. 1). The staging $\mathrm{PET} / \mathrm{CT}$ scan confirmed the tumor extension and lymph node involvement, with no distant metastasis spread (Fig. 2). At tumor board the potential treatment related risks were discussed and was taken decision the treatment to start with induction chemotherapy, followed by radiation or chemoradiation based on treatment response. According to NPC2003-GPOH protocol three courses of induction chemotherapy with a single dose Cisplatin 80 $\mathrm{mg} / \mathrm{m}^{2}$ at dayl and continuous infusion of 1000 $\mathrm{mg} / \mathrm{m}^{2} 5$-FU every day for days 1-5 were applied, and folinic acid. The control MRI found considerable reduction of the tumor mass in all areas of involvement (Fig. 3). The control PET-CT revealed full treatment response with no metabolic active lesions and described persistence of soft tissue thickening with no metabolic activity at sphenoid sinus, left ethmoidal cells, posterior wall of left maxillary sinus (Fig. 4.).

Patient was discussed again at tumor board and based on good treatment response of induction chemotherapy and severe hematological toxicity was decided the treatment to continue with radiotherapy alone. The child was irradiated with VMAT technique with cone beam CT guidance (IGRT-image guided radiotherapy) to a total dose of $50.4 \mathrm{~Gy}$ in 1.8 Gy daily fractions for the nasopharynx, the bilateral regional lymph nodes levels Ib-II-III-IV-V and retropharyngeal lymph nodesand the areas of initial extension of the tumor (Fig. 5). As the initial tumor was located retrobulbary next to the left optic nerve and left eye bulb, left lens, delivering high radiation dose will increase the risk of late toxicity,and taking in account the full metabolic tumor regression at PET CT, a decision for dose deescalation to 50.4Gy was made. No additional treatment was applied. On follow up imaging 3 months post radiotherapy, another MRI and PET/CT were performed. MRI showed further reduction of the size of the tumor (Fig. 6). PET/CT confirmed the soft tissue thickening with no metabolic activity at sphenoidal sinus, left ethmoidal cells, posterior wall of left maxillary sinus, but detected solitary osteolytic bone lesions in left ischiadic bone. Further MRI of the pelvis was perform. The progression of the disease was proved 4 months post end of treatment.

\section{Discussion}

NPC in children is often diagnosed in locoregional advanced stage [1]. For best initial evaluation of tumor spread two imaging modality have shown to be of value $[6,7]$. MRI give us correct definition of $\mathrm{T}$ an $\mathrm{N}$ category, but PET/CT could early detect distant metastasis $[7,8]$. They both are used also for treatment follow up. The implementation of follow up PET/CT in the present case enabled early detectection of distant metastasis.

The preferred treatment protocol for advanced childhood NPC is the one used at the prospective, multicenter study NPC-2003-GPOH/DCOG [4, 6]. It includes 3 courses of neoadjuvant chemotherapy with cisplatin, 5-fluorouracil, and folinic acid followed by chemoradiation, andinterferon betatreatment for six months. The cumulative irradiation dose was 54 Gy inpatients with complete remission after neoadjuvant chemotherapy, and 59.4 Gy in 
not full responders. After a median follow-up of 30 months (range, 6-95 months), the reported event free survival and the overall survival are respectively $92.4 \%$ and $97.1 \%$ with no severe toxicity. These are the best reported results but still many answers are there to be answered. If reduction of the dose in good responders is suitable for all patients, or couldthe concomitant chemotherapy be omitted and further reduce the rate ofearly and late morbidity. Randomize trials are needed.

NPC-2003-GPOH/DCOG study together with two more were the first exploring dose deescalation and proved that is feasible [4-6]. The total radiation dose used earlier were in the range of 66-70Gy at a price of high toxicity [1]. For further reduction of toxicity advanced radiotherapy techniques as IMRT [9] and his advanced type VMATand IGRT are in use. IMRT and VMAT enable precisely delivery of the maximum dose only at the target with simultaneous decrease the dose at organs at risk. And IGRT with cone beam computer tomography imaging in treatment room help us every day to visualize the target volume and not to miss the target, so accurate delivery. The combination of VMAT and IGRT could further contribute for improving tumor control and reducing toxicity.

Investigation of Epstein Barr virus is recommended as it could serve as prognostic and predictive marker [6].

With the present paper a high-risk childhood undifferentiated NPC is presented. Diagnostic MRI and $\mathrm{PET} / \mathrm{CT}$ were performed with good concordance for primary tumor extension and lymph node involvement. At presentation and no distant metastasis were detected. Three coursesinduction chemotherapy were applied according to NPC2003-GPOH protocolwith good treatment response. The restaging PET/CT found no distant metastasis. Deescalation protocol radiotherapy alone was delivered to 50.4Gy total dose with IGRT VMAT irradiation technique. No interferon beta was applied.At three month PET/CT follow up a solitary bone lesion was detected.

The reason for distant failure could be explained be the advance stage, the low radiation dose compared to 70Gy, no concomitant chemotherapy with radiotherapy or the lack of interferon beta treatment. The weakness of the treatment is the lack of Epstain Barr viral infection evaluation. Although radiation dose was comparably low an excellent local control was achieved. The lack of concomitant chemotherapy could be a better explanation for distant failure, but it was not applied because of the potential hematological toxicity, which was observed in the patient with radiotherapy alone. This case shows the importance or PET/CT like imaging modality for follow up for early detection of distant failure. MRI still keeps it role for best imaging modality for locoregional evaluation of the tumor spread.

\section{Conclusion}

The present case proved that in high risk patients more aggressive treatment strategies should be recommended with no omission of concurrent chemotherapy even after full response. Investigation for Epstein Barr virus infection is mandatory and IFN beta treatment should be explored. Deescalation of radiotherapy probably is not appropriate in this group of patients, no matter the excellent response to induction chemotherapy. The higher late morbidity risk should be accepted in order to eradicate the disease. MRI and PET CT should be used as complementary imaging modalities for early detection of locoregional or distant metastasis. Randomize trials are warranted to answer in which patients deescalation of radiation dose and omission of concurrent chemotherapy is reasonable even with the implementation of advanced IMRT techniques like VMAT and set a certain unified international guidelines for treatment improvements.

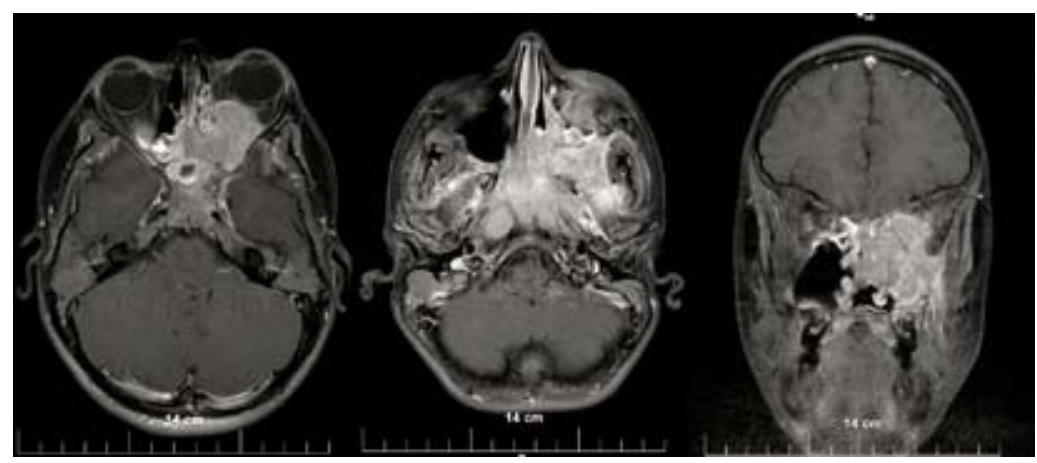

Fig. 1. Diagnostic MRI before treatment 


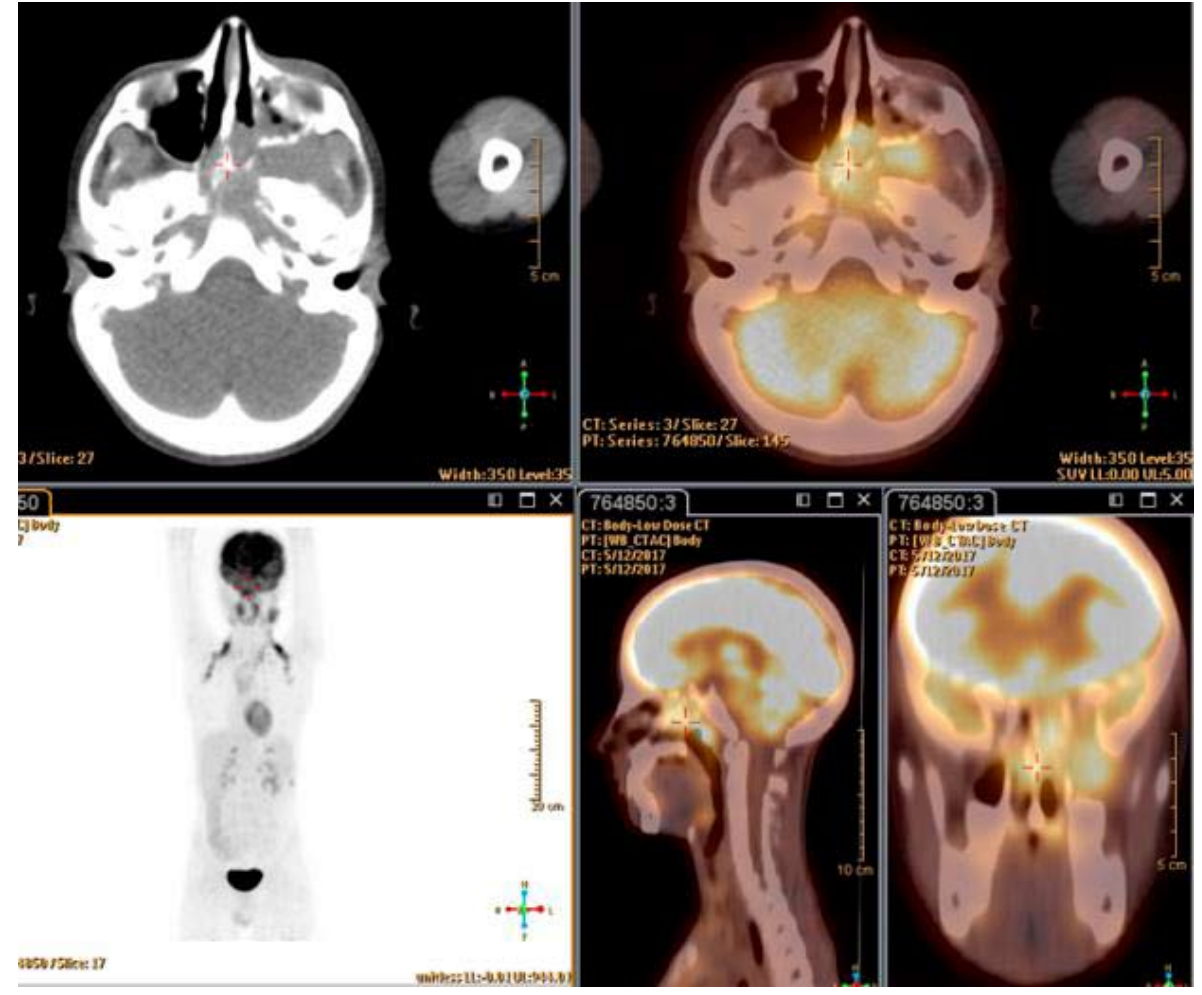

Fig. 2. Diagnostic $P E T / C T$ before treatment

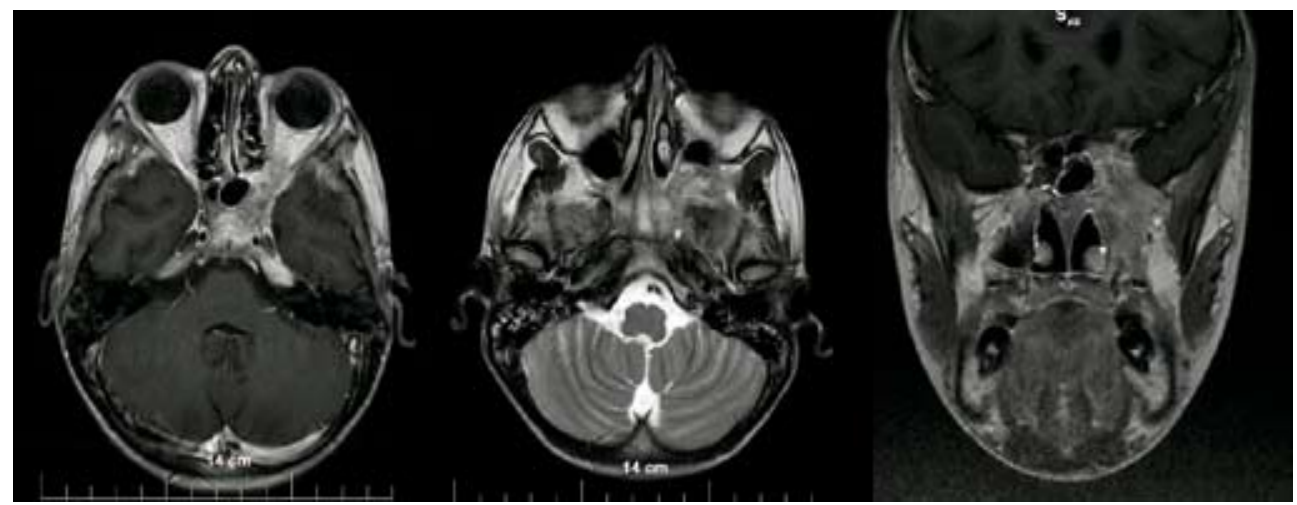

Fig. 3. Control MRI after 3 courses induction chemotherapy before radiotherapy

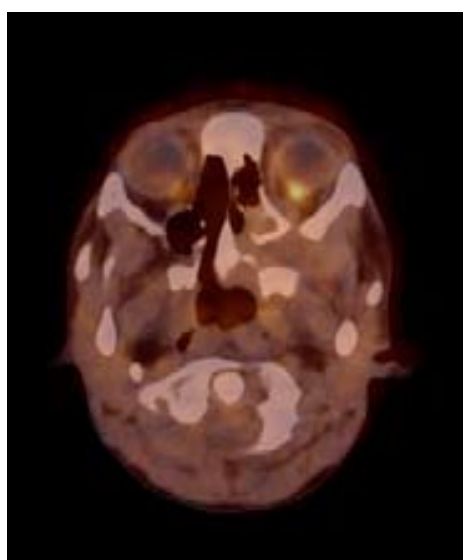

Fig. 4. Control PET/CT after 3 courses induction chemotherapy before radiotherapy 

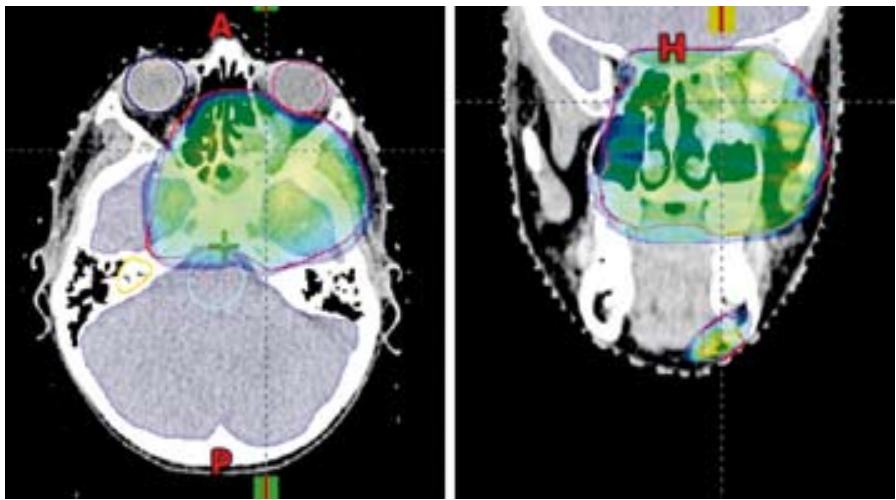

Fig. 5. VMAT radiotherapy treatment plan

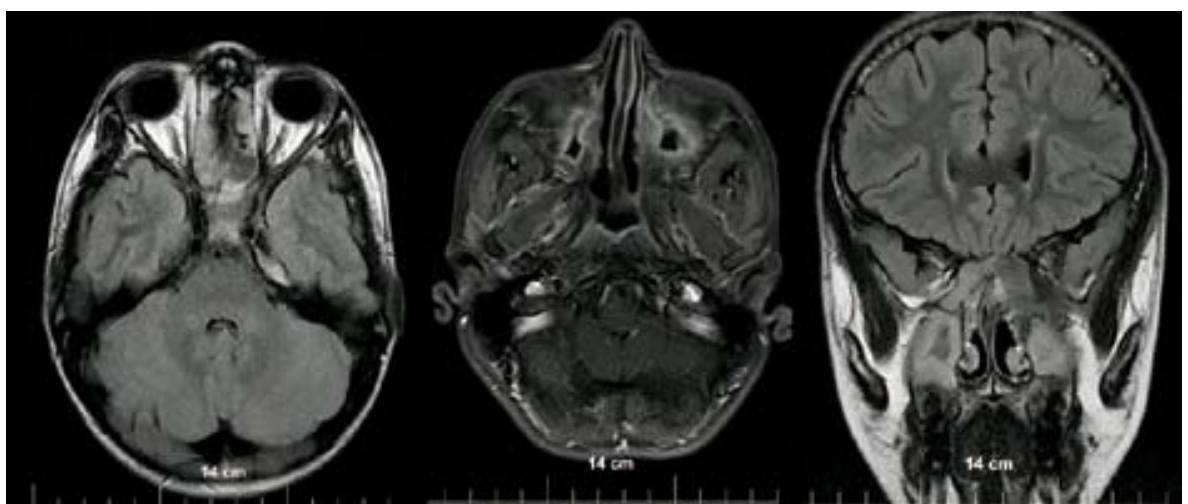

Fig. 6. Follow up MRI3 months post radiotherapy

\section{Bibliography:}

[1] Ayan I, Altun M. Nasopharyngeal carcinoma in children: retrospective review of 50 patients. Int J Radiat Oncol Biol Phys. 1996;35:485492.

[2] Valerianova Z, Atanasov T, Vukov M. et al. Cancer Incidence in Bulgaria, 2014 \& 2015. Bulgarian National Cancer Registry. Sofia, 2017

[3] Dourthe ME, Bolle S, Temam S, Jouin A, Claude L, Reguerre Y, Defachelles AS, Orbach D, Fresneau B. Childhood Nasopharyngeal Carcinoma: State-of-the-Art, and Questions for the Future. J Pediatr Hematol Oncol. 2018 Mar;40(2):85-92

[4] Mertens R, Granzen B, Lassay L, et al. Treatment of nasopharyngeal carcinoma in children and adolescents: definitive results of a multicenter study (NPC-91-German Society of Pediatric Oncology and Hematology ). Cancer 2005;104:1083-1089.

[5] Rodriguez-Galindo C, Wofford M, Castleberry RP, et al. Preradiation chemotherapy with methotrexate, cisplatin, 5-fluorouracil, and leucovorin for pediatric nasopharyngeal carcinoma. Cancer 2005;103:850-857

[6] Buehrlen M, Zwaan CM, Granzen B, et al. Multimodal treatment, including interferon beta, of nasopharyngeal carcinoma in children and young adults: preliminary results from the prospective, multicenter study NPC-2003-GPOH/DCOG. Cancer. 2012;118:4892-4900

[7] Kontny U, Franzen S, Behrends U, et al. Diagnosis and treatment of nasopharyngeal carcinoma in children and adolescents-recommendations of the GPOH-NPC study group. Klin Padiatr. 2016;228:105-112

[8] Cheuk DKL, Sabin ND, Hossain M, et al. PET/CT for staging and follow-up of pediatric nasopharyngeal carcinoma. Eur J Nucl Med Mol Imaging. 2012;39:1097-1106

[9] Guo Q, Cui X, Lin S, Lin J, Pan J Locoregionally advanced nasopharyngeal carcinoma in childhood and adolescence: Analysis of 95 patients treated with combined chemotherapy and intensity-modulated radiotherapy.Head Neck. 2016 Apr;38 Suppl 1:E665-72. 